\title{
Self-Consistent Chaos in the Beam-Plasma Instability
}

\author{
J.L. Tennyson ${ }^{*}$ \\ Stanford Linear Accelerator Center \\ Stanford University \\ Palo Alto, CA 94305 \\ J.D. Meiss \\ Program in Applied Mathematics \\ Box 526, University of Colorado \\ Boulder CO 80309 \\ P.J. Morrison \\ Department of Physics and \\ Institute for Fusion Studies \\ The University of Texas at Austin \\ Austin, Texas 78712
}

DOE/ET/53088--587

February 8, 1993

\begin{abstract}
The effect of self-consistency on Hamiltonian systems with a large number of degreesof-freedom is investigated for the beam-plasma instability using the single-wave model of O'Neil, Winfrey, and Malmberg. The single-wave model is reviewed and then rederived within the Hamiltonian context, which leads naturally to canonical actionangle variables. Simulations are performed with a large $\left(10^{4}\right)$ number of beam particles interacting with the single wave. It is observed that the system relaxes into a time asymptotic periodic state where only a few collective degrees are active; namely,
\end{abstract}

- Posthumous. Prepared by J.D.M. and P.J.M.

$$
\begin{aligned}
& 1 \text { Heftal }
\end{aligned}
$$

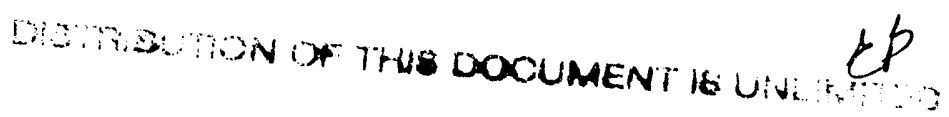


a clump of trapped particles oscillating in a modulated wave, within a uniform chaotic sea with oscillating phase space boundaries. Thus self-consistency is seen to effectively reduce the number of degrens-of-freedom. A simple low degree-of-freedom model is derived that treats the clump as a single macroparticle, interacting with the wave and chaotic sea. The uniform chaotic sea is modeled by a fluid waterbag, where the waterbag boundaries correspond approximately to invariant tori. This low degree-offreedom model is seen to compare well with the simulation. 


\section{Introduction}

Chaotic motion in Hamiltonian systems is common whenever there are more than one degreeof-freedom [1]. Often, the systems studied are low-dimensional approximations of many degree-of-freedom systems. In some cases, such as planetary dynamics, highly accurate descriptions can be given with only a few degrees-of-freedom. However, there are many situations, such as galactic dynamics, where the number of degrees-of-freedom is essentially infinite. Generally, one expects suck systems to exhibit greater chaos when the dimension increases; furthermore, some effects such as "Arnold diffusion" are possible only with a larger number of degrees-of-freedom. Even in high dimensional cases it is often of interest to study a low dimensional approximation, for example to study the motion of a single star in a given galactic gravitational potential - this was the motivation for the classic study of Hénon and Heiles (see references in [1]). Such an approximation is not self-consistent. Other wellstudied examples of this type include the motion of charged particles in electromagnetic fields, where the fields produced by the particles are ignored; the motion of tracer particles in a fluid, where the influence of these particles on the fluid velocity field is ignored (the passive advection problem); and the so-called "kinematic" dynamo, where a velocity field can intensify a magnetic field, but the back-reaction of the field is ignored.

There has been little work on the effect of self-consistency. In this paper we show how it is possible in a system with a large number of degrees-of-freedom for the inclusion of self-consistency to result in dynamics with "effectively" few degrees-of-freedom.

The model considered here treats self-consistency in the simplest possible way. A large number of noninteracting particles is influenced by a time-dependent potertial. The Hamiltonian for each particle has one and one half degrees-of-freedom, and so the motion can be chaotic. However, each of the particles is charged and therefore contributes to the 
potential - this is the self-consistent effect. In the model, we assume that the field has a single degree-of-freedom: i.e., that the spatial dependence of the field is given. Thus each particle experiences forces due to the others, but only to the extent that the other particles contribute to the single mode of the field. This is in contrast to the fully self-consistent $n$ body dynamics, where each particle interacts directly with every other particle. This latter case is considerably more difficult.

Models similar to the one described above may be appropriate for many physical situations; for example, a galaxy with a predominantly axisymmetric gravitational potential that is perturbed by a small number of modes, say those corresponding to spiral density waves. Each star contributes to these modes, and also has a possibly chaotic motion in the corresponding field. Similar effects occur for planetary rings, beam-beam interactions in accelerators, tearing mode-plasma interactions in tokamaks, etc.

- The specific problem we consider is the beam-plasma instability. The formulation is due to O'Neil, Wirfrey and Malmberg (hereafter referred to as $O W M$ ) [2]. Here a beam of charged particles moves in a background neutral plasma. The system is unstable to the formation of electrostatic plasma waves. Following [2] we suppose that the most unstable of these waves predominates, and neglect the remainder of the modes. OWM showed that the wave grows in amplitude until it traps the beam particles. It then saturates and begins to oscillate in amplitude as the beam particles slosh in the wave potential. In a later study Mynick and Kaufman [3] developed a simple model to account for these oscillations - they assumed that the beam could be represented by a rigid bar in phase space. When the beam is trapped in the wave, the rigid bar begins to rotate. Mynick and Kaufman computed the frequency shift and amplitude oscillations of the wave as it interacts with this rigid bar. Smith and Pereira [4] noted that since the amplitude of the plasma wave oscillates, the beam particles can experience chaotic motion. They studied the motion of a test particle in a model of this oscillating field and showed that much of the test particle phase space is 
indeed chaotic. However, there is an island in the phase space where the motion is regular; they noted that some fraction of the beam particles in the numerical experiments of OWM should find themselves in the correct region of phase space to be trapped in this oscillating island. Later Adam, Laval and Mendonca [5] studied a model in which a single bunch of particles, what we will call a macroparticle, interacts with the plasma wave. As we will show below using the Hamiltonian formulation, this two degree-of-freedom system is integrable due to the existence of a phase symmetry. In [5] it was shown that the macroparticle system has solutions which correspond to periodic oscillations of the bunch in the wave.

In Sec. 2 we review the derivation of the OWM model, deriving the Hamiltonian formulation of [3] directly from the Vlasov-Poisson equations. Sec. 3 discusses numerical solutions of the OWM equations with up to $10^{5}$ beam particles. The simulations show that the linear instability saturates and oscillates about a finite amplitude for at least 100 periods; as far as we can determine, the oscillations persist and the system becomes asymptotically periodic. We study the motion of a single test particle in this periodic potential, showing that a substantial portion of the original beam is indeed trapped in a stable island in the test particle phase space. However more than half the beam finds itself in the chaotic region of phase space, and spreads more or less uniformly over this region. The upper and lower boundaries of this "chaotic sea" are formed from invariant tori of the test particle system.

In Sec. 4 we construct a four degree-of-freedom model. One degree-of-freedom is the wave, the second corresponds to the trapped bunch of beam particles - the macroparticle, and the last two describe the chaotic sea. These two degrees-of-freedom describe the oscillations of the boundary of the chaotic sea and are derived from the "waterbag" approximation. A waterbag consists of a constant phase space density between two moving boundaries. In our case the simulations show that the phase space density of the chaotic particles is indeed nearly constant and the boundaries of the chaotic zone are formed from invariant surfaces well outside the oscillating separatrix of the wave. We model these boundaries with sinusoidal 
curves, an assumption consistent with that of the single mode in the potential. Finally, the frequency shift of the trappe 1 rarticle oscillations due to the chaotic sea is computed analytically from this model.

\section{Single Wave Model}

O'Neil, Winfrey, and Malmberg (OWM) [2] introduced the single wave model to describe the growth and saturation of the weak beam-plasma instability. In this section we briefly review the approximations made in their derivation, rederive the model within the Hamiltonian context and obtain the Hamiltonian form previously presented by Mynick and Kaufman [3], discuss linear instability, and finally consider a special case where only a single beam particle is included.

\subsection{Derivation}

To obtain the single wave model, the response of the plasma and beam to the fields are treated separately. We consider only the one-dimensional, collisionless, nonrelativistic, electrostatic case. The total electron density

$$
n(x, t)=n_{p}(x, t)+n_{b}(x, t)=n_{p}(x, t)+\sum_{j=1}^{N} \delta\left(x-x_{j}(t)\right)
$$

is a sum of contributions from the plasma and from a set of $N$ beam particles at positions $x_{j}(t)$. Beam particles evolve according to the fully conlinear force equation

$$
m \ddot{x}_{j}=-2 E\left(x_{j}, t\right)
$$

where the electron charge is $-e$.

A basic assumption of the model is that the phase velocity of the resulting instability is much larger than the velocities of particles in the background plasma: the plasma responds non-resonantly, and trapping effects of plasma particles in the wave can be neglected. This 
implies that the plasma responds approximately linearly to the wave so that we can define a linear hermetian dielectric operator $\bar{\epsilon}$ such that

$$
4 \pi e n_{p}(x, t)=(1-\hat{\epsilon}) \varphi^{\prime \prime}(x, t)
$$

where $\varphi$ is the electrostatic potential, $E=-\varphi^{\prime}$. Substituting this into Poisson's equation for $\varphi$ yields

$$
\hat{\epsilon} \varphi=4 \pi e n_{b}(x, t) .
$$

For a homogeneous plasma, $\widehat{\epsilon}$ is a convolution operator in both $x$ and $t$, and Poisson's equation is most easily treated by Fourier transform. In this representation the dielectric becomes a real function, $\epsilon(k, \omega)$. For a weak beam, the right-hand side of eq. (4) is "small" implying that the electrostatic response is dominated by the zeros, $\epsilon\left(k, \omega_{0}(k)\right)=0$, of the dielectric. It is a reasonable approximation to expand $\epsilon$ about one such zero retaining only the first derivative of $\epsilon$ with respect to $\omega$ :

$$
(k, \omega) \approx \epsilon\left(k, \omega_{0}\right)+\left.\frac{\partial}{\epsilon} \partial \omega\right|_{\omega=\omega_{0}}\left(\omega-\omega_{0}\right)=\epsilon^{\prime}\left(\omega-\omega_{0}\right) .
$$

For example, for a cold plasma $\epsilon=1-\omega_{p}^{2} / \omega^{2}$, and $\partial \epsilon /\left.\partial \omega\right|_{\omega=\omega_{0}} \equiv \epsilon^{\prime}=2 / \omega_{p}$. Transforming back to the time dornain and using eq. (4) then gives

$$
\dot{E}_{k}+i \omega_{0} E_{k}=\frac{4 \pi e}{k L \epsilon^{\prime}} \sum_{j=1}^{N} e^{-i k x,(t)},
$$

where we have used the Fourier transform of the beam density of eq. (1)

$$
n_{b}(k, t)=\frac{1}{L} \int_{0}^{L} d x e^{-i k x} n_{b}(x, t)=\frac{1}{L} \sum_{j=1}^{N} e^{-i k x,(t)}
$$

with $L$ defining the periodicity length of the system.

At this point we assume that the electrostatic field has only one spatial Fourier component, i.e., that there is a single wave. It is expected that the single wave approximation is appropriate when the width of the unstable spectrum in $k$-space is relatively narrow in units 
of $2 \pi / L$. In this case, if $k$ represents the most unstable mode, the amplitude of all other Fourier components will be exponentially smaller than the single wave during the linear growth stage. Of course, some time after nonlinear saturation of the instability, the other modes will become important. As is well known, the width of the unstable spectrum depends on the small parameter $\left(n_{b} / n_{p}\right)^{1 / 3}$, so that the single wave model will be most appropriate in the weak beam case.

Under the single wave assumption eq. (2) yields

$$
\begin{aligned}
& \dot{x}_{j}=p_{j} / m \\
& \dot{p}_{j}=-e\left(E_{k} e^{i k x_{j}}+E_{-k} e^{-i k x}\right) .
\end{aligned}
$$

Equation (8) together with eq. (6) are the closed dynamical system that governs the interaction of a single wave with the beam particles.

\subsection{Hamiltonian Structure and Derivation}

Now consider the derivation of the equations of motion, eqs. (6) and (8), within the Hamiltonian context. The derivation proceeds by first considering the kinematics, i.e. the dynamical variables used to describe the state of the system, and then the dynamics, obtained by finding the appropriate Hamiltonian.

We begin the first part by supposing that the electrons are described by specifying their phase space coordinates $\left(x_{j}, p_{j}\right)$, where $j=1,2, \ldots M$. The first $N(<M)$ of these particles is singled out to represent the beam dynamics, while the remaining $M-N$ particles represent the background plasma. The phase space density, i.e. distribution function, can be represented as follows:

$$
\begin{aligned}
f(x, p, t) & =f_{b}(x, p, t)+f_{p}(x, p, t) \\
& =\sum_{j=1}^{N} \delta\left(x-x_{j}(t)\right) \delta\left(p-p_{j}(t)\right)+\sum_{j=N+1}^{M} \delta\left(x-x_{j}(t)\right) \delta\left(p-p_{j}(t)\right) .
\end{aligned}
$$


The Poisson bracket in terms of $\left(x_{j}, p_{j}\right)$, where $j=1,2, \ldots M$, has the standard canonical form

$$
[g, h]=\sum_{j=1}^{M}\left(\frac{\partial g}{\partial x_{j}} \frac{\partial h}{\partial p_{j}}-\frac{\partial h}{\partial x_{j}} \frac{\partial g}{\partial p_{j}}\right) \equiv[g, h]_{b}+[g, h]_{p},
$$

where $g$ and $h$ are functions defined on phase space.

It is desired to describe the state of system in terms of the above phase space coordinates for the beam particles. However, for the background plasma, the phase space coordinates of these particles will be replaced by a Vlasov type distribution function, $f_{p}$. This can be achieved by mapping the Poisson bracket of eq. (10) to these variables; but $f_{p}$, unlike $\left(x_{j}, p_{j}\right)$, is not a canonically conjugate set of coordinates, i.e. $f_{p}$ is a noncanonical variable, therefore the resulting Poisson bracket possesses noncanonical form [6]. In order to effect this transformation the chain rule for functional derivatives is required. Suppose

$$
g\left(x_{j}, p_{j}\right)=G\left[f_{p}\right]
$$

where $j=N+1 . V+2, \ldots M$. Here $G\left[f_{p}\right]$ is a functional of $f_{p}$; the relationship between the phase space function $g$ and the functional $G$ is obtained by inserting the representation defined by eq. (9) into the functional $G$. The chain rule is obtained by varying both sides of this equation:

$$
\begin{aligned}
\delta g & =\sum_{j=N+1}^{M}\left(\frac{\partial g}{\partial x_{j}} \delta x_{j}+\frac{\partial g}{\partial p_{j}} \delta p_{j}\right)=\delta G=\int \frac{\delta G}{\delta f_{p}} \sum_{i=N+1}^{M}\left(\frac{\delta f_{p}}{\delta x_{j}} \delta x_{j}+\frac{\delta f_{p}}{\delta p_{j}} \delta p_{j}\right) d x d p \\
& =\sum_{j=N+1}^{M}\left(\delta x_{j} \int\left(\frac{\delta f_{p}}{\delta x_{j}}\right)^{\dagger} \frac{\delta G}{\delta f_{p}} d x d p+\delta p_{j} \int\left(\frac{\delta f_{p}}{\delta p_{j}}\right)^{\dagger} \frac{\delta G}{\delta f_{p}} d x d p\right) .
\end{aligned}
$$

The operators $\delta f_{p} / \delta x_{j}$ and $\delta f_{p} / \delta p_{j}$ are obtained by variation of eq. (9) with respect to the plasma particles, and $\dagger$ denotes the formal adjoint. The result is finally

$$
\begin{aligned}
& \frac{\partial g}{\partial x_{j}}=\int\left(\frac{\partial}{\partial x} \frac{\delta G}{\delta f_{p}}\right) \delta\left(x-x_{j}\right) \delta\left(p-p_{j}\right) d x d p=\left.\left(\frac{\partial}{\partial x} \frac{\delta G}{\delta f_{p}}\right)\right|_{(x, p,)} \\
& \frac{\partial g}{\partial p_{j}}=\int\left(\frac{\partial}{\partial p} \frac{\delta G}{\delta f_{p}}\right) \delta\left(x-x_{j}\right) \delta\left(p-p_{j}\right) d x d p=\left.\left(\frac{\partial}{\partial p} \frac{\delta G}{\delta f_{p}}\right)\right|_{\left(x_{j}, p_{j}\right)} .
\end{aligned}
$$


Insertion of eq. (13) into the second term of eq. (10) yields the bracket

$$
[G, H]=\int f_{p}\left\{\frac{\delta G}{\delta f_{p}}, \frac{\delta H}{\delta f_{p}}\right\} d x d p+\sum_{j=1}^{N}\left(\frac{\partial G}{\partial x_{j}} \frac{\partial H}{\partial p_{j}}-\frac{\partial H}{\partial x_{j}} \frac{\partial G}{\partial p_{j}}\right)
$$

where

$$
\{g, h\} \equiv \frac{\partial g}{\partial x} \frac{\partial h}{\partial p}-\frac{\partial h}{\partial x} \frac{\partial g}{\partial p} .
$$

Here the quantities $G$ and $H$ are functionals of $f_{p}$, but according to eq. (9) they can be thought of as ordinary functions of the beam particle coordinates $\left(x_{j}, p_{j}\right)$ where $j=$ $1,2, \ldots N$.

The Hamiltonian for a hybrid system composed of the beam electrons coupled to the background Vlasov pla.ma electrons is obtained by inserting

$$
f(x, p, t)=f_{p}(x, p, t)+\sum_{j=1}^{N} \delta\left(x-x_{j}(t)\right) \delta\left(p-p_{j}(t)\right)
$$

into the following energy expression:

$$
H[f]=\frac{m}{2} \int v^{2} f d x d p+\frac{1}{8 \pi} \int E^{2} d x
$$

This results in

$$
H\left[f_{p} ; x_{j}, p_{j}\right]=\frac{1}{2 m} \int p^{2} f_{p} d x d p-\frac{e}{2} \int f_{p} \varphi_{p} d x+\sum_{j=1}^{N}\left(\frac{p_{j}^{2}}{2 m}-e \varphi_{p}\left(x_{j}\right)-\frac{e}{2} \varphi_{b}\left(x_{j}\right)\right),
$$

where $\varphi_{p}\left(x_{j}\right)$ and $\varphi_{b}\left(x_{j}\right)$ are the contributions to the electrostatic potential of the plasma and beam charge, respectively. The Hamiltonian of eq. (18) together with the bracket of eq. (14) yields the hybrid system.

Now we can turn to the task of obtaining, from the hybrid system, the approximate system of Sec. 2.1. First, the background plasm; s assumed to be described by an equilibrium distribution function of compact support in velocity, plus the single linear wave, whose phise velocity is outside the equilibrium support. In this way wave-particle effects are eliminated in the background plasma. Upon inserting the wre perturbation i the distribution function, the analysis of [7] and [8] implies that the linearization of the plasma energy becomes 
identically the well-known expression for the dielectric energy of a plasma wave. Second, the self-interaction potential of the beam, $\varphi_{b}$, is neglected in comparison to that of the plasma, $\varphi_{p}$, a justifiable assumption in light of smallness of $n_{b} / n_{p}$. Thus, eq. (18) becomes

$$
H\left(E_{k}, E_{-k}, x_{j}, p_{j}\right)=\frac{L}{4 \pi} \omega_{0} \epsilon^{\prime}\left|E_{k}\right|^{2}+\sum_{j=1}^{N}\left(\frac{p_{j}^{2}}{2 m}-\frac{i e}{k} E_{k} e^{i k x}+\frac{i e}{k} E_{-k} e^{-i k x}\right) .
$$

It remains to find the appropriate Poisson bracket in terms of $E_{k}$ and $E_{-k}$ instead of $f_{p}$. Since the plasma is in essence being modeled as a fluid, an easy way to obtain this is to map the first term of eq. (14) to fluid variables, a transformation that is easily effected by incorporating techniques similar to those used in obtaining eq. (14), and then transforming to the variables $E_{k}$ and $E_{-k}[9]$. This results in

$$
[g, h]=\sum_{j=1}^{N}\left(\frac{\partial g}{\partial x_{j}} \frac{\partial h}{\partial p_{j}}-\frac{\partial h}{\partial x_{j}} \frac{\partial g}{\partial p_{j}}\right)-\frac{i}{L} \frac{4 \pi}{\epsilon^{\prime}}\left(\frac{\partial g}{\partial E_{k}} \frac{\partial h}{\partial E_{-k}}-\frac{\partial h}{\partial E_{k}} \frac{\partial g}{\partial E_{-k}}\right) .
$$

The bracket of eq. (20) with eq. (19) produces eqs. (6) and (8) in the form

$$
\dot{E}_{k}=\left[E_{k}, H\right], \quad \dot{x}_{j}=\left[x_{j}, H\right], \quad \dot{p}_{j}=\left[p_{j}, H\right]
$$

The bracket of eq. (20) is not quite canonical; however, with the substitution

$$
E_{k}=-i\left[\frac{4 \pi}{L \epsilon^{\prime}}\right]^{1 / 2} \mathcal{J}^{1 / 2} e^{-i \vartheta} \quad E_{-k}=i\left[\frac{4 \pi}{L \epsilon^{\prime}}\right]^{1 / 2} \mathcal{J}^{1 / 2} e^{i \vartheta}
$$

it assumes canonical form

$$
[g, h]=\sum_{j=1}^{N}\left(\frac{\partial g}{\partial x_{j}} \frac{\partial h}{\partial p_{j}}-\frac{\partial h}{\partial x_{j}} \frac{\partial g}{\partial p_{j}}\right)+\left(\frac{\partial g}{\partial \vartheta} \frac{\partial h}{\partial \mathcal{J}}-\frac{\partial h}{\partial \vartheta} \frac{\partial g}{\partial \mathcal{J}}\right),
$$

while the Hamiltonian of eq. (19) becomes

$$
H\left(\vartheta, \mathcal{J}, x_{j}, p_{j}\right)=\omega_{0} \mathcal{J}+\sum_{j=1}^{N}\left[\frac{p_{j}^{2}}{2 m}-\frac{2 e}{k}\left(\frac{4 \pi}{L \epsilon^{\prime}}\right)^{\frac{1}{2}} \mathcal{J}^{\frac{1}{2}} \cos \left(k x_{j}-\vartheta\right)\right] .
$$

To complete the derivation, it is convenient to introduce scaled, dimensionless variables based on the fundamental frequency,

$$
\omega_{b}^{3}=\frac{4 \pi e^{2} N}{m L \epsilon^{\prime}} .
$$


Here $\omega_{b}$ is a harmonic mean of the beam's plasma frequency and $1 / \epsilon^{\prime}$, which is of order the background plasma frequency. Note that the small parameter $\left(n_{b} / n_{p}\right)^{1 / 3}$ is represented by the ratio $\omega_{b} / \omega_{0}$. By a sequence of time-dependent canonical transformations and scalings, the Hamiltonian of eq. (24) becomes

$$
H\left(J, \theta, p_{j}, \xi_{j}\right)=\sum_{j=1}^{N}\left[\frac{1}{2} p_{j}^{2}-2\left(\frac{J}{N}\right)^{\frac{1}{2}} \cos \left(\xi_{j}-\theta\right)\right],
$$

where the dimensionless variables are defined by

$$
\tau=\omega_{b} t, \quad \xi_{j}(\tau)=k x_{j}-\omega_{0} t, \quad J=\frac{k^{2}}{m \omega_{b}} \mathcal{J}, \quad \theta \equiv \vartheta-\omega_{0} t .
$$

Thus in terms of the new canonically conjugate pair, $(\theta, J)$, the fast frequency, $\omega_{0}$, has been eliminated. The new position variable, $\xi_{j}$, which is canonically conjugate to $p_{j}$, is defined in a frame moving at the phase velocity $\omega_{0} / k$. This Hamiltonian was (in essence) previously discovered (but not derived) by Mynick and Kaufman [3].

It is often convenient to use a noncanonical wave amplitude variable instead of actionangle variables. This is easily done if we use as independent variables the amplitude $\Phi$ and its complex conjugate $\Phi^{*}$ defined by

$$
\Phi(\tau)=\left(\frac{J}{N}\right)^{\frac{1}{2}} e^{-i \theta}
$$

In these coordinates the Poisson bracket becomes

$$
\left[\Phi^{*}, \Phi\right]=i / N
$$

and the Hamiltonian takes the form

$$
H=\sum_{j=1}^{N}\left[\frac{1}{2} p_{j}^{2}-\Phi e^{i \xi,}-\Phi^{*} e^{-i \xi}\right] .
$$

This is a natural form for the Hamiltonian since the first term is the particle kinetic energy and the last two represent the electrostatic potential energy. The equations of motion are obtained from the Poisson bracket,

$$
\frac{d \Phi}{d \tau}=[\Phi, H]=\left[\Phi, \Phi^{*}\right] \frac{\partial H}{\partial \Phi^{*}},
$$


which together with the canonical bracket for $\left(\xi_{j}, p_{j}\right)$ produces

$$
\begin{aligned}
& \frac{d \Phi}{d \tau}=\frac{i}{V} \sum_{j=1}^{N} e^{-i \xi_{j}} \\
& \frac{d^{2} \xi_{j}}{d \tau^{2}}=i \Phi e^{i \xi_{j}}-i \Phi^{*} e^{-i \xi_{j}} .
\end{aligned}
$$

Note that these equations hold for arbitrary choices of the physical parameters, such as $e / m$ and the beam dersity; it is only the relationship between scaled variables and physical variables which changes. (Of course, the single wave model is appropriate only when the beam is sufficiently weak, and such a parameter free formulation is possible only in the single wave case.)

The Hamiltonian, eq. (26), has one obvi us symmetry (we don't know of any non-obvious symmetries) which is the translation, $\xi_{j} \rightarrow \xi_{j}+\alpha, \theta \rightarrow \theta+\alpha$. This implies that the total momentum

$$
P \equiv \sum_{j=1}^{N} p_{j}+J
$$

is conserved. To take advantage of this, define the canonical transformation generated by $F_{2}=P \theta+\sum p_{j}^{\prime}(\xi,-\theta)$ which gives the new momenta, $\left(p_{j}^{\prime}=p_{j}, P\right)$, and angles, $\left(\psi_{j}=\xi_{j}-\theta, \theta\right)$. The new Hamiltonian is

$$
H\left(p_{j}, \psi_{j}\right)=\sum_{j=1}^{N}\left[\frac{1}{2} p_{j}^{2}-\frac{2}{\sqrt{N}}\left(P-\sum_{k=1}^{N} p_{k}\right)^{\frac{1}{2}} \cos \psi_{j}\right]
$$

which has effectively $N$ degrees-of-freedom since $P$ is an ignorable coordinate.

\subsection{Linear Instability}

To establish the fact that the Hamiltonian of eq. (30) properly describes at least the linear stage of the weak beam-plasma instability, consider an initial condition consisting of a coldbeam: $\xi_{j}^{0}=2 \pi j / N, p_{j}^{0}=0$, and no field, $\Phi^{0}=0$. Linearizing the Hamiltonian about this 
equilibrium, one obtains the quadratic form

$$
H=\sum_{j=1}^{\infty} \frac{1}{2} \delta p_{j}^{2}-i \delta \Phi \delta \xi_{j} e^{i \xi_{j}^{0}}+i \delta \Phi^{*} \delta \xi_{j} e^{-i \xi_{j}^{0}}
$$

The resulting linear equations of motion can be straightforwardly diagonalized to obtain the characteristic polynomial $\omega^{2 N-4}\left(\omega^{6}-1\right)=0$, where the solutions have been assumed to vary as $e^{-i \omega t}$. This has the solutions $\omega^{3}= \pm 1$, which include the standard beam-plasma instability frequencies together with their conjugate roots as required because of the Hamiltonian nature of the flow (recall that if $\omega$ is an eigenvalue then $\omega^{*},-\omega$ and $-\omega^{*}$ must also be eigenvalues). In dimensional units, using eq. (27), we have

$$
\widehat{\omega}_{j}=\omega_{b} e^{i j \pi / 3}, \quad j=0,1, \ldots, 5
$$

which the includes the unstable beam-plasma mode (the case $j=2$ ). We can physically identify the eigenmodes by considering the equations of motion. Differentiating the equation for $\Phi$ twice and substituting for $\ddot{\xi}$ gives

$$
\frac{d^{3} \delta \Phi}{d \tau^{3}}=\frac{1}{N} \sum_{j=1}^{N} e^{-i \xi_{j}^{0}} \delta \vec{\xi}_{j}=i \delta \Phi
$$

upon noting that $\sum e^{-2 i \xi_{j}^{0}}=0$. This shows that the nonzero frequencies are associated with nonzero $\Phi$. The eigenmodes for the conjugate roots, $\omega^{*},-\omega$ and $-\omega^{*}$, are the same as that for $\omega$ except for varying choices of signs. The remaining roots of the characteristic equation ( $\omega=0$ of multiplicity $2 N-4)$ have eigenmodes with $\delta \Phi=\delta \Phi^{*}=0$ and positions given by the $N-2$ independent solutions of $\sum e^{-i \xi_{j}^{0}} \delta \xi_{j}=0$. The double multiplicity of each of these roots arises from allowing the momenta to be nonzero, $\delta p_{j} \propto \delta \xi_{j}$ so the mode is rigidly translating.

As will be seen in the next section, the linear beam-plasma instability saturates by trapping the particles in the electrostatic well of the wave. 


\subsection{Single Particle Case}

The only exactly solvable case of the Hamiltonian eq. (26) appears to be that of a single particle. Use of the conservation of total momentum, eq. (33), reduces this case to one effective degree-of-freedom, and it can be integrated by quadrature. When $N$ is larger than unity we expect to lose integrability. It will be of interest later to consider the case when there are $N_{m}$ particles clumped together, and we temporarily ignore the remaining particles. We call the clumped particles a macroparticle. In this case, eq. (26) becomes

$$
H=\frac{p^{2}}{2 N_{m}}-2 N_{m}\left(\frac{J}{N}\right)^{\frac{1}{2}} \cos (\xi-\theta),
$$

where $p=N_{m} p_{1}=N_{m} p_{2} \ldots$ is the macroparticle momentum. The Hamiltonian $H$ can be reduced to one degree-of-freedom by defining the total momentum $P=p+J$ as before to obtain

$$
H=\frac{p^{2}}{2 N_{m}}-2 N_{m}\left(\frac{P-p}{N}\right)^{\frac{1}{2}} \cos \psi .
$$

The equations for this case were studied in detail by Adam, Laval and Mendonca [5], who did not use the Hamiltonian approach.

There are, in general, three nondegenerate fixed points of the motion implied by eq. (39). These occur at the points defined by

$$
p_{0}^{3}-p_{0}^{2} P+N_{m} \frac{N_{m}}{N}=0, \quad \psi_{0}=0 \quad \text { or } \pi .
$$

The fixed point with $\left(p_{0}<0, \psi_{0}=0\right)$ is stable and corresponds to the macroparticle sitting in the bottom of the potential well. The two fixed points with $\left(p_{0}>0, \psi=\pi\right)$ are less intuitive. These exist only if $P>3 N_{m}\left(\frac{N_{m}}{4 N}\right)^{1 / 3}$. They correspond to a particle perched on the top of the potential well. The lower momentum particle is unstable, while the larger momentum particle is actually stable. This can be seen in fig. 1 which shows contours of constant $H$ in the $(p, \psi)$ phase space. Small oscillations about the stable fixed point are such 
that as the particle starts to fall off the potential hill, the wave phase shifts so as to catch the particle.

The final "fixed point" is the degenerate case $(p=P, \psi=$ arbitrary) for which the wave amplitude is zero. This corresponds to the unstable equilibrium from which the beam plasma instability results, as discussed in the previous section.

Small oscillations about these fixed points, with $\left(J_{0}=P-p_{0}\right)$ are governed by the linearized Hamiltonian

$$
\begin{aligned}
\delta^{2} H & =\frac{\delta p^{2}}{2 M_{e}}+N_{m}\left(\frac{J_{0}}{N}\right)^{\frac{1}{2}} \delta \psi^{2} ; \\
M_{e} & \equiv N_{m} \frac{2 J_{0}}{2 J_{0}-p_{0}} .
\end{aligned}
$$

Here $M_{e}$ is the effective mass due to the self-consistent coupling. Note that since $p_{0}<0$, the effective mass is smaller than that of a particle in a fixed potential well. This gives a bounce frequency of

$$
\omega_{B}^{2}=2\left(\frac{J_{0}}{N}\right)^{\frac{1}{2}}\left(1-\frac{p_{0}}{2 J_{0}}\right) .
$$

The first factor is just the bounce frequency of a particle in a fixed well, normalized in accord with eq. (27). The latter term provides an increase in the frequency, as also discussed in [3].

To compare these with the simulations in the next section, we set the total momentum, $P$, to zero, corresponding to the initial conditions $J(0)=p(0)=0$. In this case $J(\tau)=-p(\tau)$ and there is only one fixed point

$$
\Omega \equiv \frac{p_{0}}{N_{m}}=-\left(\frac{N_{m}}{N}\right)^{1 / 3}, \quad \psi=0 .
$$

From the simulations we will find $N_{m} \simeq .4 N$, and thus that

$$
\begin{gathered}
\Omega=-0.74 \\
|\Phi|=0.29 \\
\omega_{B}=0.94 .
\end{gathered}
$$




\section{Simulations}

Simulations of the model of eqs. (32) were carried out for several initial conditions and a number of different integration algorithms. The results shown below were obtained with a symplectic, leap frog method.

\subsection{OWM Model}

In the simulations the particles are initialized as a cold beam with uniform spacing $\xi_{j}=$ $2 \pi j / N, j=0, \ldots N$, and zero momentum $p_{j}=0$. The initial wave amplitude is set to be very small. In fig. 2 , the wave amplitude is shown as a function of time for eq. (32) with $N=10^{4}$. For small $\tau$ the wave amplitude grows exponentially in time at the rate predicted by eq. (36) and at the phase velocity $v_{\phi}=\mathcal{R}\left(e^{2 \pi i / 3}\right)=-0.5$. and ours is. As the wave grows, the beam experiences a growing sinusoidal perturbation, and as can be seen in the density plot of fig. 3, the beam density also varies sinusoidaly. Near $\tau=16$ the beam curls over as the particles begin to oscillate in the wave. Consequently, the amplitude of the wave reaches a maximum.

At this stage the beam density, $n_{b}(\xi, \tau)$, develops cusps at the positions where the beam curls over; see fig. 3. Note that though there are many spatial harmonics in the beam density, the single wave model does not allow the development of similar harmonics in the potential. These would lead to the growth of other waves and undoubtedly greatly change the subsequent behavior of the system.

Nonetheless, the subsequent development of the OWM dynamics is quite interesting. As the beam particles begin to oscillate in the wave, their oscillation frequencies depend upon their energy, just as for a single particle in a fixed potential. Thus as the beam begine to rotate about the potential minimum, those particles closer to the center have larger oscillation frequencies than those near the "separatrix." 
If the wave amplitude were fixed, one would see phase mixing of the particles (visualized as an ever tighter spiral in the particle phase space), and the oscillations in the particle total energy would damp away - this is the mechanism of Landau damping in a large amplitude wave discussed by $\mathrm{O}^{\prime} \mathrm{Neil}[10]$.

However since $v_{\phi} \approx-0.5$ and the beam is initialized at $v=0$, when the beam particles oscillate in the wave, their net mornentum also oscillates. Hence, because of the conservation of total momentum, eq. (33), the wave momentum, $J$, must also oscillate as well. Therefore the wave amplitude is not fixed and each beam particle experiences an oscillating potential. As is well known, the phase space for a single beam particle in a given oscillating potential has chaotic zones, especially for the regions near the oscillating separatrix. In the beam particle phase space, fig. 4, we show the phase space positions of each of the beam particles at a fixed time. Note the two distinct regions: one chaotic in appearance with a nearly uniform density, and the other a more coherent cluster of particles. In the cluster one still sees evidence of the initial beam, though it has wrapped around a number of times.

In the simulations, which were carried out up to $\tau \approx 650$, the oscillations in the potenti 1 persisted, and indeed, as can be seen in fig. 2 the oscillations become increasingly periodic with lime. Furthermore as we varied $N$ up to $10^{5}$ and the changed integration accuracy, we noted that these oscillations became more periodic and constant in amplitude as the number of particles increased and as the accuracy improved. Thus we believe that the asymptotic state is a periodic one.

Meanwhile, the particle phase space exhibits quite complicated behavior. About $60 \%$ of the particles - those with relatively large energies in the wave frame - experience chaotic motion, and spread out roughly uniformly in a region of phase space whose average width is $\Delta \omega=4.7$. We called the chaotic particles the "stochastic sea" in 1983 - a more modern terminology is the "chaotic sea". The remaining $40 \%$ of the particles are clustered tightly together in a clump which has a width of order $\pi / 2$ and a density about 6 times larger than 
the background, recall fig. 3. These particles continue to oscillate coherently in the wave, as seen in the sequence of phase space pictures, fig. 5. Also shown in the figures is the instantaneous separatrix of the wave - that is the separatrix that a single beam particle would see in a fixed potential at the current amplitude. Note that wave amplitude and the momentum of the cluster oscillate $180^{\circ}$ out of phase. This clump of particles is treated as a single particle, the macroparticle, in the model of Sec. 4.

In addition to the uniform cold beam, several different initial conditions have been partially studied. For example we started particles on a circular ring, $\xi^{2}+p^{2}=r^{2}$, and also considered a bunched beam with particles placed uniformly in a limited range, $-1<\xi<1$ with $p \neq 0$. In these cases some subset of the particles remained coherently bunched as a macroparticle, and the system did not appear to settle into an equilibrium. We have not investigated this in any generality and do not know the extent of the initial conditions which will give rise to a periodic final state.

\subsection{Test Particle}

To investigate further dynamics of the beam particles, consider the "test particle" motion of a single particle in a given oscillating potential. These are obtained from the nonselfconsistent, one and one-half degree-of-freedom Hamiltonian

$$
H_{t}(p, \xi, \tau)=\frac{1}{2} p^{2}-2\left(\frac{J(\tau)}{N}\right)^{\frac{1}{2}} \cos (\xi-\theta(\tau)),
$$

where $J$ and $\theta$ are considered to be given periodic functions of $\tau$. This system has been studied in the same context by Smith and Pereira [4], who used a simple model for $J(\tau)$ and $\theta(\tau)$.

Here we determine $J$ and $\theta$ numerically, from the simulations of Sec. 3.1, building these functions from an average over a number of periods of the oscillations.

A stroboscopic plot of the test particle dynamics is shown in fig. 6 for several different values of $\theta$. The dots represent the trajectories of a number of different test particles. As was 
also noted in [4], there is a prominent stable island in the test particle phase space which oscillates exactly out of phase with the potential; much of the rest of the phase space is chaotic. Also shown in fig. 6 are the corresponding plots of the beam particle phase space here of course each point in the plots is the position of one of the 10,000 beam particles. Note that the macroparticle clump sits, as near as can be ascertained, at the position of the test particle island. This verifies an assertion in [4], where it was merely noted that some fraction of the beam particles initially stretched across the position of the test particle island.

\section{Chaotic Sea Model}

As we have seen from the simulations, the asymptotic state of the cold beam initial condition, evolved under the OWM Hamiltonian, appears to be almost exactly periodic. Approximately $40 \%$ of the initial beam forms the clump of particles that oscillates in the potential well formed by the wav:. The remaining particles spread out approximately uniformly in phase space between two oscillating boundaries, which are approximately invariant surfaces of the test particle Hamiitonian $H_{t}$.

In this section we use these ideas to obtain a reduced Hamiltonian model of four degreesof-freedom, which approximately describes the full 10,001 degree-of-freedom system. In the model, as noted above, we assume that the clump of regularly oscillating particles is localized enough so that all these particles can be treated as one located at $(\xi, p)$. This macroparticle contains $N_{m}$ particles and hence a mass $m N_{m}$ and charge $-e N_{m}$. The approximation that the $N_{m}$ particles can be treated as a single particle ignores any internal degrees-of-freedom of the cluster (of which there are $N_{m}-1$ ). It is clear that a correction to the model would include, at the least, some sort of rotational degree-of-freedom; this would be similar to the analysis of Mynick and Kaufman [3] who assumed that the cluster of particles formed a "bar" in phase space (an assumption that is reasonable only for the first few trapping oscillations). 
In our case the particles in the macroparticle could be thought of as rotating on the invariant surfaces of the test particle Hamiltonian, eq. (45), about the central periodic orbit. This behavior could be modeled by the addition of an nscillator degree-of-freedom to the system; however, we neglect this motion here.

Much more interesting is the treatment of the chaotic particles. As noted in Sec. 3.1, the phase space density of these particles appears to be nearly constant up to fairly sharp boundaries in velocity space. We assume that these particles can be treated as a continuum with a constant phase space density $f_{c}$, between the boundaries $v_{+}(x, t)$ and $v_{-}(x, t)$; thus,

$$
n_{c}(x, t)=\int_{v_{-}}^{v_{+}} f_{c} d v=f_{c}\left(v_{+}-v_{-}\right)
$$

is the density of the chaotic sea. The total number of such particles in the length $L$ will be denoted by $N_{c}=N-N_{m}$. Particles in the chaotic sea evolve according to eq. (2), and hence $f_{c}$ evolves according to the Vlasov equation. As is well known, and easy to see, the Vlasov equation in this situation can be exactly reduced to two equations for the evolution of the boundaries [11]. These equations are called the waterbag equations:

$$
\begin{aligned}
& \frac{\partial v_{+}}{\partial t}+v_{+} \frac{\partial v_{+}}{\partial x}=-e E \\
& \frac{\partial v_{-}}{\partial t}+v_{-} \frac{\partial v_{-}}{\partial x}=-e E .
\end{aligned}
$$

Following the philosophy of the derivation of the OWM model, where only a single wavenumber in $\varphi$ is kept, we retain only one wavenumber in $v_{ \pm}(x)$ :

$$
v_{ \pm}=v_{ \pm}^{0}+\tilde{v}_{ \pm} e^{i k x}+\tilde{v}_{ \pm}^{*} e^{-i k x}
$$

The equations of motion then become

$$
\left(\frac{\partial}{\partial t}+i k v_{ \pm}^{0}\right) \tilde{v}_{ \pm}=-e E_{k}
$$

where $v_{ \pm}^{0}$ are the mean velocities (which do not change in time according to eq. (47)). Note that this implies that the boundary of the chaotic sea, an invariant surface of $H_{t}$, is sinusoidal 
in shape. We see from fig. 5 that this is reasonable since the boundary deviation is small and it is not too close to the "separatrix" of the wave.

The evolution of the field is obtained irom Poisson's equation, as previously, except that the density of the chaotic sea, as given by eq. (46), must be included. Thus eq. (6) becomes

$$
\dot{E}_{k}+i \omega_{0} E_{k}=\frac{4 \pi e}{k \epsilon^{\prime}}\left[f_{c}\left(\tilde{v}_{+}-\tilde{v}_{-}\right)+\frac{N_{m}}{L} e^{-i k x_{m}} .\right]
$$

These equations are nondimensionalized, using eq. (27); in addition defining

$$
\omega_{ \pm} \equiv \frac{k v_{ \pm}^{0}-\omega_{0}}{\omega_{b}}, \quad V_{ \pm} \equiv \frac{k}{\omega_{b}} \tilde{v}_{ \pm} e^{i \omega_{0} t}
$$

In terms of these variables the equations of motion become

$$
\begin{aligned}
\dot{\Phi} & =i \frac{N_{c}}{N \Delta \omega}\left(V_{+}-V_{-}\right)+i \frac{N_{m}}{N} e^{-i \xi}, \\
\ddot{\xi} & =i \Phi e^{i \xi}-i \Phi^{*} e^{-i \xi}, \\
\dot{V}_{ \pm} & =-i \omega_{ \pm} V_{ \pm}+i \Phi,
\end{aligned}
$$

where $\Delta \omega=\omega_{+}-\omega_{-}$is the average, nondimensional width of the chaotic sea.

This set of equations is also a Hamiltonian system, with the wave action-amplitude variables defined in eq. (28), and the new action-amplitude variables for the chaotic sea defined by

$$
V_{+}=\left(\frac{J_{+} \Delta \omega}{N_{c}}\right)^{\frac{1}{2}} e^{-i \theta_{+}}, \quad V_{-}=\left(\frac{J_{-} \Delta \omega}{N_{c}}\right)^{\frac{1}{2}} e^{+i \theta_{-}},
$$

which results in the Poisson bracket relations

$$
\left[V_{ \pm}, V_{ \pm}\right]= \pm i \frac{\Delta \omega}{N_{c}}
$$

The Hamiltonian takes the form

$$
\left.H=\frac{p^{2}}{2 N_{m}}+\frac{N_{c} \omega_{+}}{\Delta \omega}\left|V_{+}\right|^{2}-\frac{N_{c} \omega_{-}}{\Delta \omega}\left|V_{-}\right|^{2}-\frac{N_{c}}{\Delta \omega} \Phi^{*}\left(V_{+}-V_{-}\right)+N_{m} \Phi^{*} e^{-i \xi}+\text { c.c. }\right\} .
$$


In terms of canonical variables this becomes

$H=\frac{p^{2}}{2 N_{m}}+\omega_{+} J_{+}-\omega_{-} J_{-}-2 \alpha\left[\sqrt{J J_{+}} \cos \left(\theta-\theta_{+}\right)-\sqrt{J J_{-}} \cos \left(\theta+\theta_{-}\right)\right]-2 \beta \sqrt{J} \cos (\theta-\xi)$.

where the coefficients are given by

$$
\alpha=\left(\frac{N_{c}}{N \Delta \omega}\right)^{1 / 2}, \quad \beta=\frac{N_{m}}{\sqrt{N}} .
$$

The first three terms in the Hamiltonian represent the total particle kinetic energy as a sum of macroparticle energy and harmonic terms for the oscillations of the chaotic sea boundary. The last three terms give the electrostatic interaction energy.

Thus we have reduced the 10,001 degree-of-freedom Hamiltonian to one of four degreesof-freedom, which describes well the motion in the periodic final state of the simulations, provided the three parameters $\omega_{+}, \omega_{-}$and $N_{m}$ are given.

\subsection{Symmetry, Equilibrium, and Stability}

The chaotic sea Hamiltonian conserves the total momentum, given by

$$
P=J+p+J_{+}-J_{-}+\frac{N_{c}}{2}\left(\omega_{+}+\omega_{-}\right) .
$$

Here the first two terms represent the momentum in the wave and macroparticle, and the last three are the contributions of the chaotic sea. These latter terms include the momentum in the oscillations of the waterbag boundary, $J_{+}-J_{-}$, and the mean momentum in the chaotic sea represented by the last term in eq. (58). This equation can also be derived from eq. (33) by splitting off the contribution of the particle momentum for the $N_{c}$ particles in the chaotic sea, treating them as a Vlasov fluid in the waterbag.

As far as we know, there are no other conservec quantities besides the energy. Thus the system has three effective degrees-of-freedom, and should exhibit the full complexity of such systems, including chaotic motion and Arnold diffusion. 
The stable equilibrium of eq. (56) corresponding to the macroparticle sitting in the potential minimum is given by the equations

$$
\begin{aligned}
\Omega \equiv \frac{p}{V_{m}} & =\dot{\xi}=\dot{\theta}=\dot{\theta}_{+}=-\dot{\theta}_{-} \\
\Omega & =\alpha^{2}\left(\frac{1}{\Omega-\omega_{+}}-\frac{1}{\Omega \cdot-\omega_{-}}\right)-\frac{\beta}{\sqrt{J}} \\
J_{ \pm} & =\frac{\alpha^{2}}{\left(\Omega-\omega_{ \pm}\right)^{2}} J .
\end{aligned}
$$

From the simulation results of fig. 5 , we found $N_{m} \approx 0.4 N, \omega_{+} \approx 1.9$, and $\omega_{-} \approx-2.8$. This gives $\alpha=.36, \beta=40$, and $\Delta \omega=4.7$. For these parameters we can solve eq. (59) to obtain

$$
\begin{aligned}
\Omega & =-0.66, \quad|\Phi|=0.73 \\
\left|V_{+}\right| & =0.29, \quad\left|V_{-}\right|=0.35 .
\end{aligned}
$$

On the other harid, using fig. 5 to determine the average phase velocity of the wave in the simulations we obtain $\Omega \approx-0.67$. The average value of $|\Phi|$ from fig. 2 is 0.75 . Both of these values certainly agree with eq. (60) more closely than they do with the single particle equilibrium eq. (44). In fact, if we take the measured value of $\Omega$ as given, then eq. (59) gives the macroparticle fraction as $42 \%$ and $|\Phi|$ becomes 0.74 , both of which are consistent with our estimates from the numerics.

To compute the frequency of small oscillations about the equilibrium, we first eliminate the action $J$ using the conservation law (58), defining phases $\psi=\theta-\xi$, and $\psi_{ \pm}=\theta_{ \pm} \mp$ $\theta$, conjugate to the momenta $p$ and $J_{ \pm}$respectively. Upon linearization the Hamiltonian becomes

$$
\delta^{2} H=\frac{1}{2} \delta \mathbf{p} \cdot \mathbf{M}^{-1} \cdot \delta \mathbf{p}+\frac{1}{2} \delta \boldsymbol{\psi} \cdot \mathbf{K} \cdot \delta \boldsymbol{\psi},
$$

where $\delta \mathbf{p}=\left(\delta p, \delta J_{+}, \delta J_{-}\right)$and $\delta \boldsymbol{\psi}=\left(\delta \psi, \delta \psi_{+}, \delta \psi_{-}\right)$are the deviations from equilibrium. The matrix $\mathbf{M}$, the effective mass matrix, is positive definite. The matrix $\mathbf{K}$, the effective 
spring constant matrix, turns out to be diagonal. In order that the three terms in $\mathbf{K}$ be positive, we must assume that $\psi=\psi_{+}=0$, while $\psi_{-}=\pi$. This is consistent with the fact that the lower boundary of the chaotic sea is observed to have a $180^{\circ}$ phase lag with respect to the upper boundary.

The fruquencies of small oscillation are given by the square roots of eigenvalues of the matrix $\mathbf{K M}^{-1}$. For the parameters of the simulation, the mass matrix is diagonal to a good approximation. The element $M_{11}^{-1}$ turns out to be identical to $1 / M_{e}$ of eq. (41); neglecting terms of order $J_{ \pm} / J$, the other diagonal elements are

$$
M_{22}^{-1}=-\frac{1}{2} \frac{\Omega-\omega_{+}}{J_{+}}, \quad M_{33}^{-1}=\frac{1}{2} \frac{\Omega-\omega_{-}}{J_{-}} .
$$

The matrix $\mathbf{K}$ is

$$
\mathbf{K}=\operatorname{dia}\left(2 \beta \sqrt{J}, \quad 2 \alpha \sqrt{J J_{+}}, \quad 2 \alpha \sqrt{J J_{+}}\right) .
$$

Using the values obtained before for the equilibrium, we determined the eigenvalues numerically from the fuli matrix. The three oscillation frequencies are

$$
\omega_{B}=(1.33,2.61,2.11)
$$

The first of these corresponds to the large oscillations observed in the simulations. The eigenfunction of this mode corresponds primarily to the $\delta p$ degree-of-freedom that describes oscillation of the macroparticle. From fig. 2 we measure the frequency of oscillations of the asymptotic state, obtaining $\omega_{B}=1.28$ - again a good agreement with the calculated value. We have no evidence for the excitation of the other two normal modes; however, it might be possible to determine these through careful simulation.

Thus we conclude that the chaotic sea model agrees with the simulations extremely well, and much better than the single particle calculation, eq. (44). 


\section{Conclusions}

We have studied the long time dynamics of the electrostatic interaction of many particles with a plasma wave. The wave arises from an instability (the beam-plasma instability) of the initial state corresponding to a cold beam of particles. In the simulations, the asymptotic state corresponds to a periodically oscillating wave amplitude together with a trapped clump of particles. About $42 \%$ of the particles are trapped by approximate invariant surface within the oscillating wave, while the remaining particles move chaotically - becoming successively trapped and detrapped.

We modeled this motion by a four degree-of-freedom Hamiltonian system, where one degree-of-freedom corresponds to the clump, two to the chaotic sea, and one to the wave. This model quantitatively captures the asymptotic state of the effectively infinite degree-offreedom system.

One would like to speculate that there are other physical systems for which the effect of self-consistency would be similar. For example, in the case of galactic dynamics, the selfconsistent propagation of a density wave would lead to chaotic dynamics for many stars, but there could be a set of stars which are coherently interacting with the wave.

As usual, a number of open questions remain:

- The periodic asymptotic state appears to arise for a number of different initial conditions in the OWM model. What is the "basin" of initial conditions which lead to such a state? One coula, for example, consider a warm beam initial state, or widely different initial states such as those discussed at the end of Sec. 3.1.

- Is there a way of self-consistently calculating the quartities $N_{m}$, and $\omega_{ \pm}$, which we took from the simulations, for the chaotic sea model?

- For the single particle Hamiltonian, there is a second stable equilibrium corresponding 
to the particle at rest at the maximum of the potential. Is there a periodic state of the many particle system. near this equilibrium?

- In the OWM model. or ly a single Fourier harmonic of the electrostatic field is kept. What is the effect of adding additional harmonics?

- The chaotic sea Hamiltonian should exhibit the full array of possible Hamiltonian motions. Are there many particle states that correspond to these motions?

\section{Acknowledgments}

Work on this project began in 1982 when all the authors were at the Institute for Fusion Studies. Due to subsequent dispersal of the authors, the paper was not completed until now. Support was provided by the U.S. Dept. of Energy Contract no. DE-FG05-80ET-53088 to The University of Texas at Austin. 


\section{References}

[1] R.S. MacKay and J.D. Meiss, Hamiltonian Dynamical Systems: a Reprint Selection, (Adam-Hilgar, 1987).

[2] T.M. O'Neil, J.H. Winfrey, and J.H. Malmberg, Nonlinear interaction of a small cold beam and a plasma, Phys. Fluids 14 (1971), 1204-1212.

[3] H.E. Mynick and A.N. Kaufman, Soluble theory of nonlinear beam-plasma interaction, Phys. Fluids 21 (1978), 653-663.

[4] G.R. Smith and N.R. Pereira, Phase-locked particle motion in a large amplituded plasma wave, Phys. Fluids 21 (1978), 2253-2262.

[5] J.C. Adam, G. Laval, and I. Mendonca, Time-dependent nonlinear Langmuir waves, Phys. Fluids 24 (1981) 260-267.

[6] P.J. Morrison, The Maxwell-Vlasov equations as a continuous Hamiltonian system, Phys. Lett. A 80 (1980), 383-386.

[7] P.J. Morrison and D. Pfirsch, Dielectric energy versus plasma energy, and Hamiltonian action-angle variables for the Vlasov equation, Phys. Fluids B 4 (1992), 3038-3057.

[8] B. Shadwick and P.J. Morrison, Neutral modes and negative energy modes, preprint, Institute for Fusion Studies Report, IFSR\# 546 (1993) University of Texas at Austin.

[9] C. Kueny, Ph.D thesis, Nonlinear instability and chaos in plasma wave-wave interactions, The University of Texas at Austin, 1993.

[10] T.M. O'Neil, Collsionless damping of nonlinear plasma oscillations, Phys. Fluids 8 (1965), 2255-2262. 
[11] R.C. Davidson, Methods of Nonlinear Plasma Theory, (Academic, New York, 1972), pp. $45-47$. 


\section{Figure Captions}

1. Contours of $H$, eq. (39), in the single particle phase space $(p, \psi)$. Units of $p$ (and $P$ ) are $N_{m}\left(\frac{N_{m}}{N}\right)^{1 / 3}$. In the upper figure $P=0$ and there is one fixed point; in the lower figure $P=2$ and there are three fixed points.

2. Plot of $|\Phi(\tau)|$, the normalized wave amplitude, for $N=10,000$ particles initialized as a cold beam.

3. Plot of the beam density as a function of position. The sinusoidal distortion of the density due to the growing wave is shown in (a) at $\tau=12.6$. By $\tau=69.3$, in (b), about 10 bounces have occurred and the macroparticle has formed, represented here by the sharp peak around $\xi=0$. The remaining, chaotic sea particles have a sinusoidal density variation.

4. Plot of the beam particle phase space at $\tau=641$ showing a well-defined macroparticle and chaotic sea.

5. Sequence of beam phase space plots over one bounce period. Note that the macroparticle bounces coherently in the wave, and the wave amplitude and chaotic sea boundaries also oscillate periodically.

6. Phase space plots comparing the full simulation of Sec. 3 with the dynamics of a test particle in a given time-dependent potential $\Phi(\tau)$ as determined by the simulation. Shown are several test particle initial conditions at three different times during a cycle. 


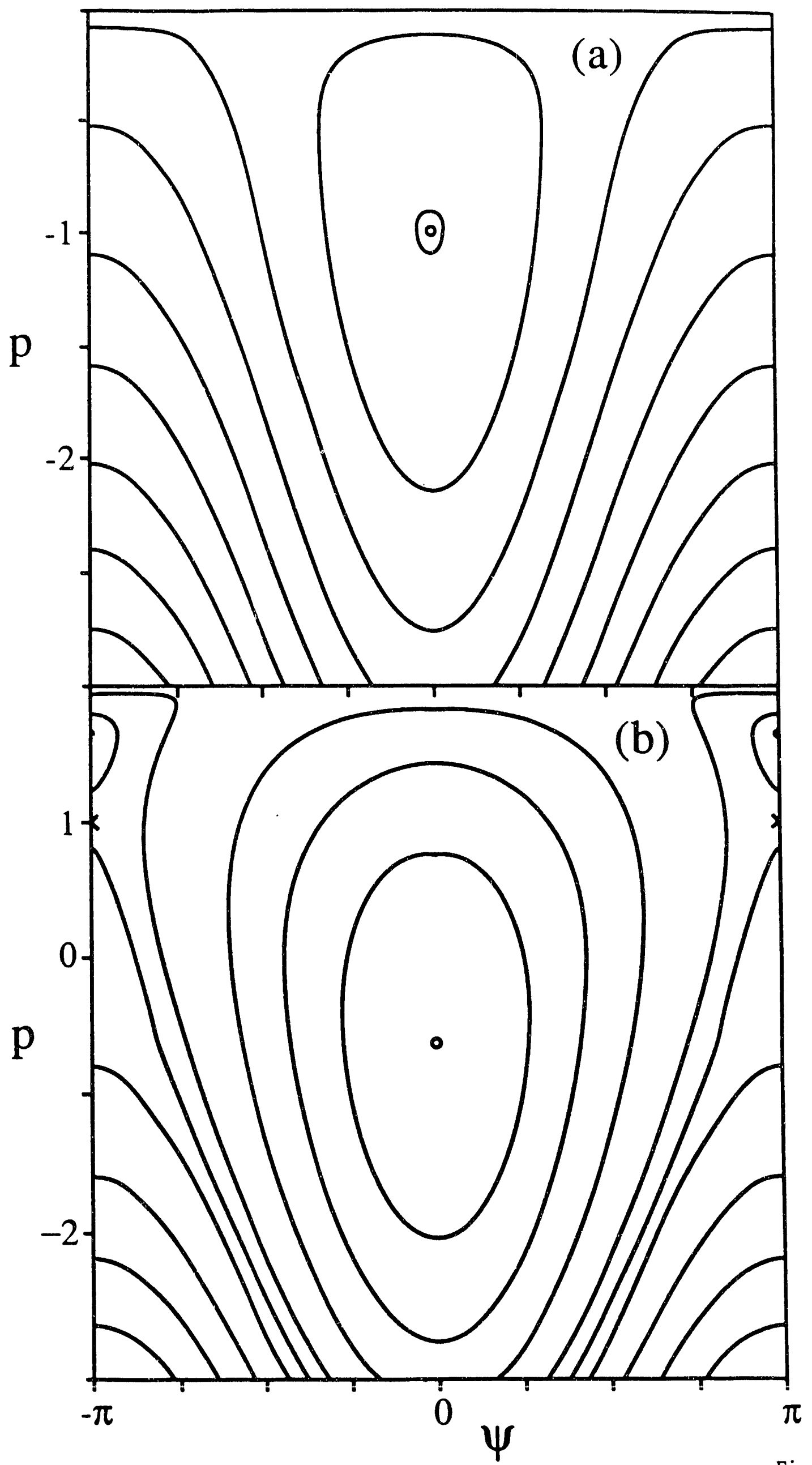

Fig. 1 


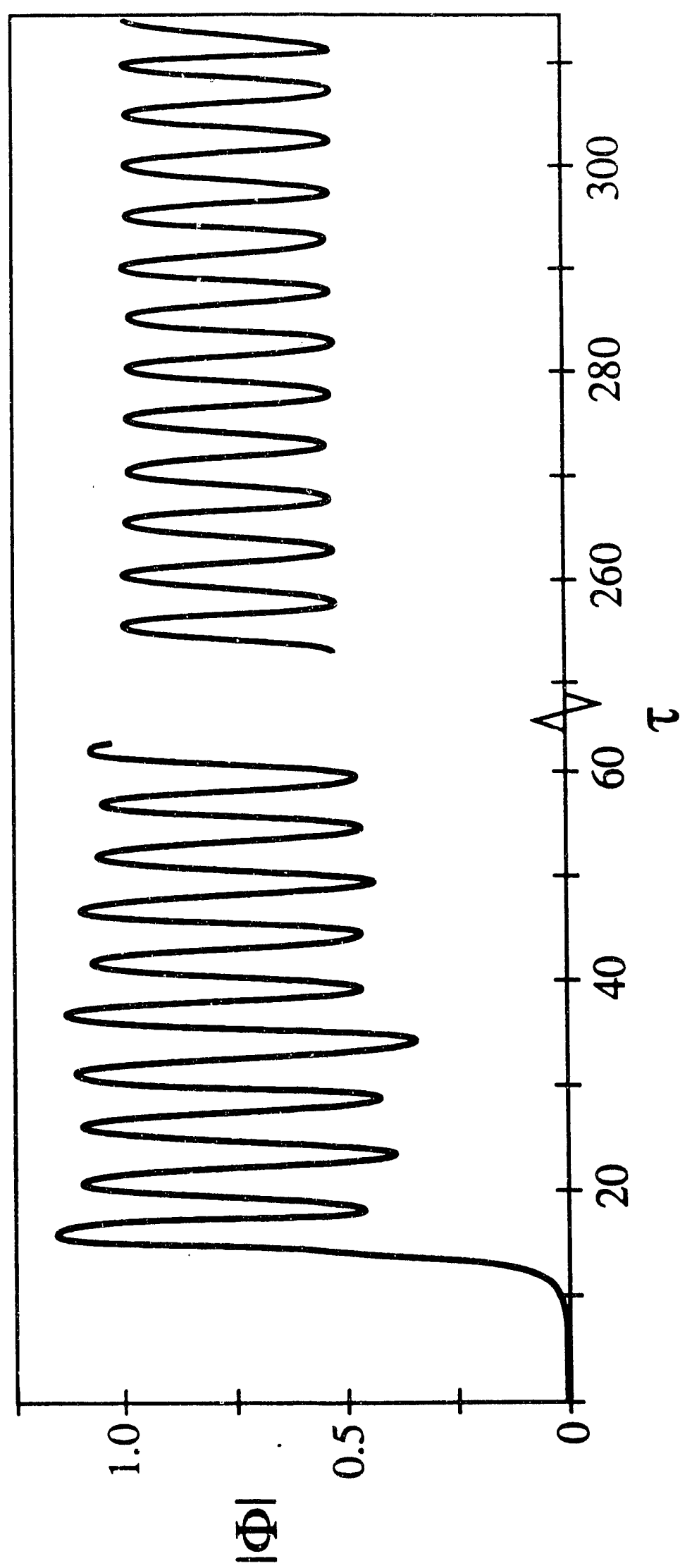

Fig. 2 


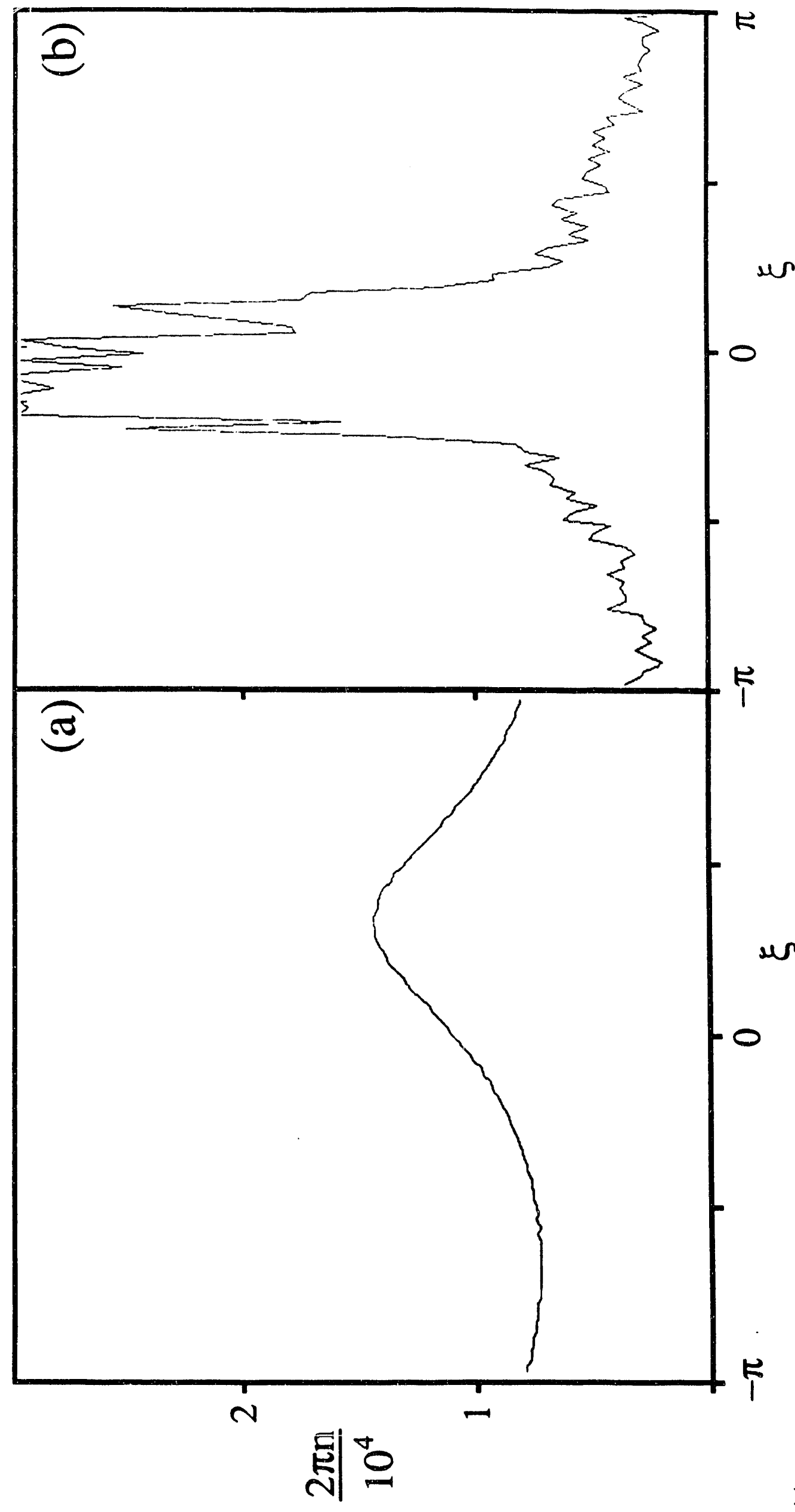

Fig. 3 


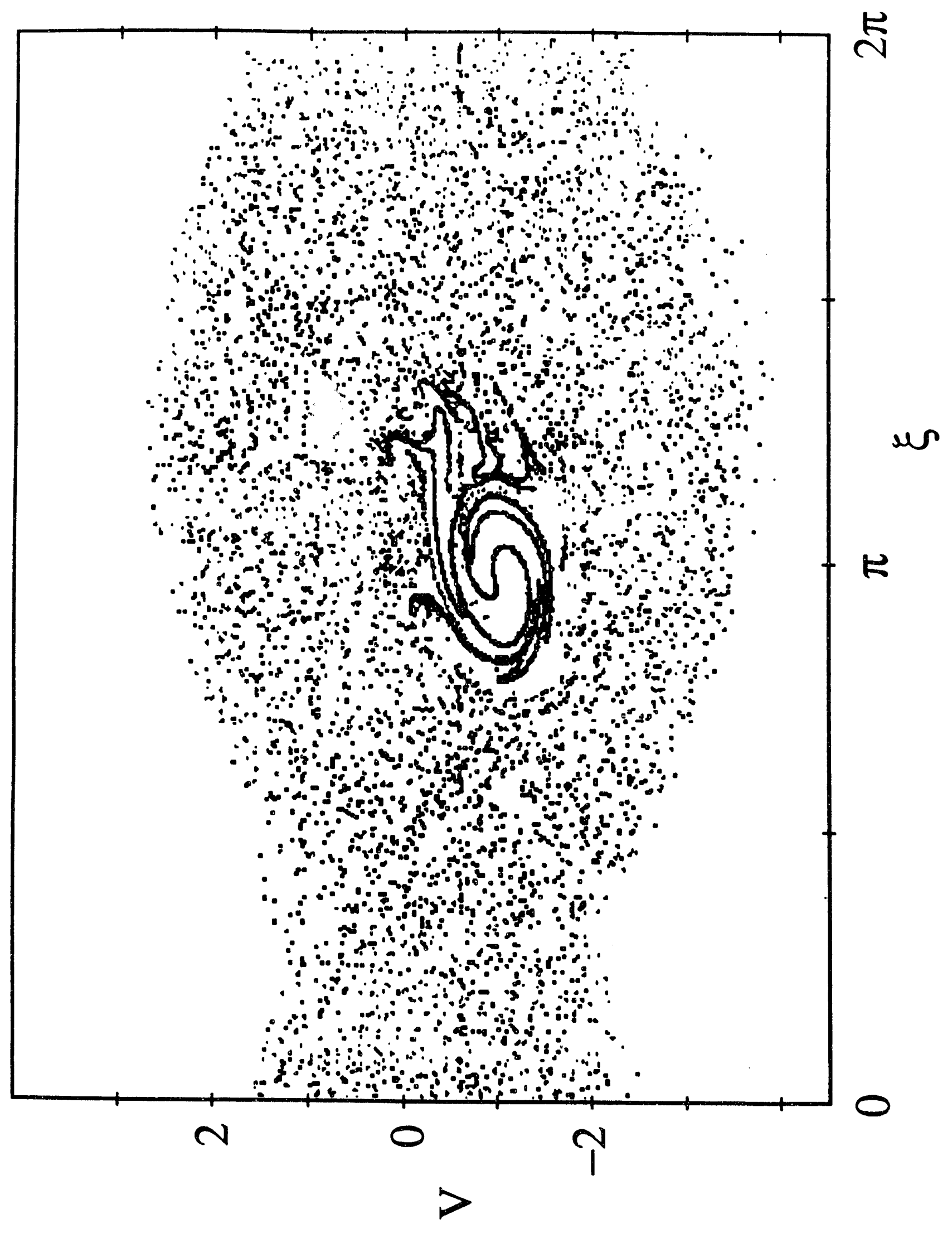

Fig. 4 


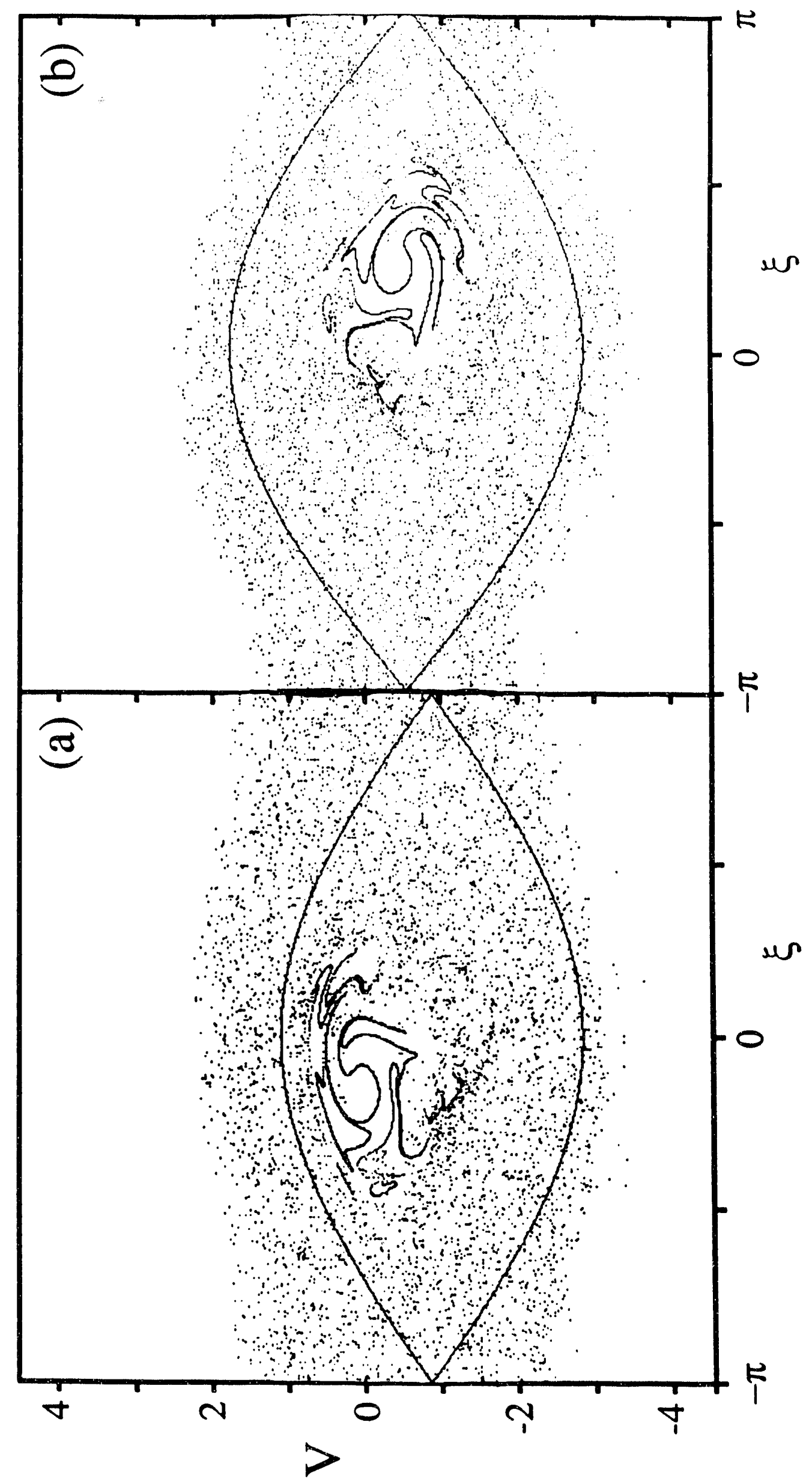

Fig. $5 a-b$ 


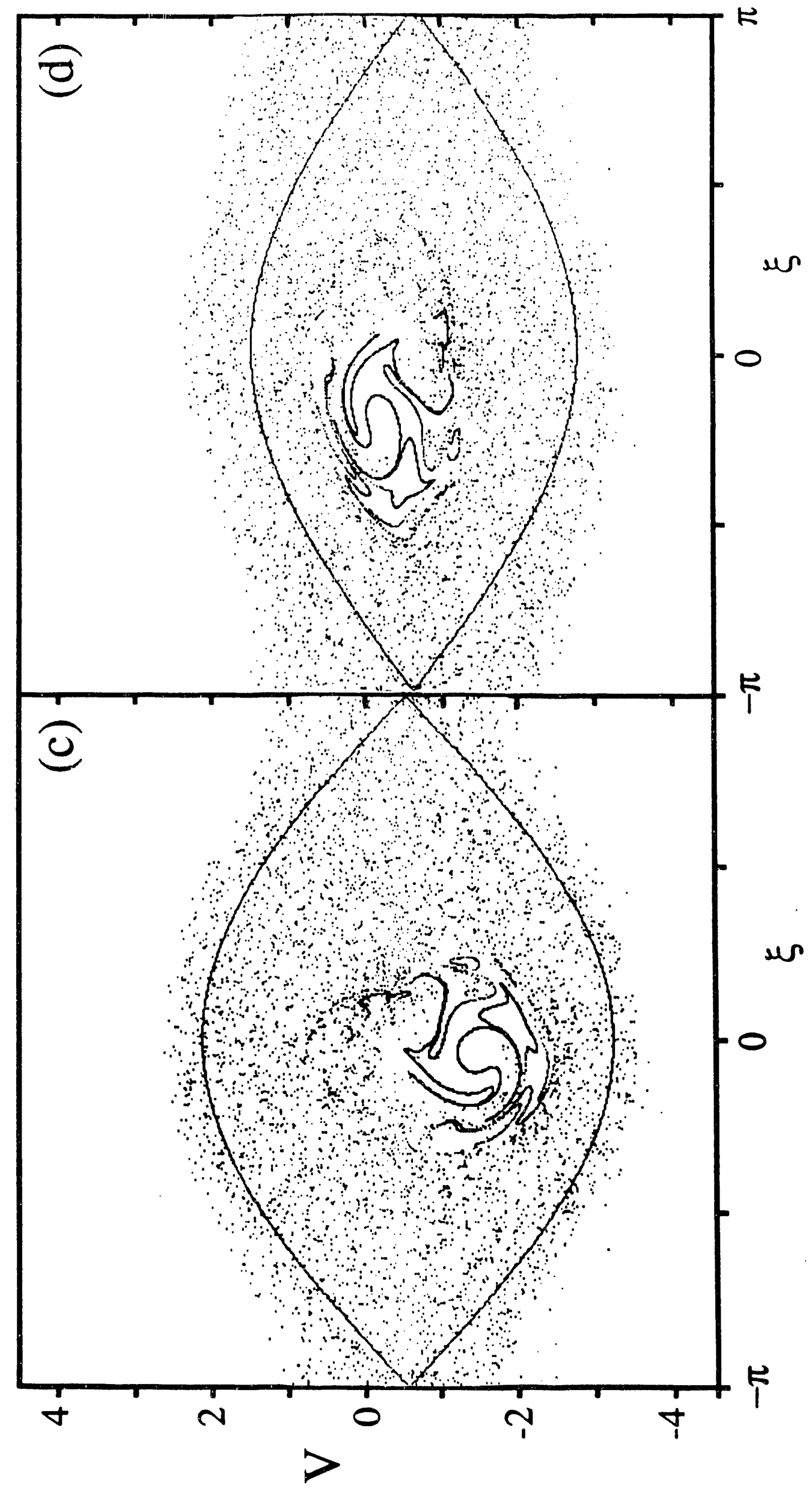

Fig. $5 c-d$ 

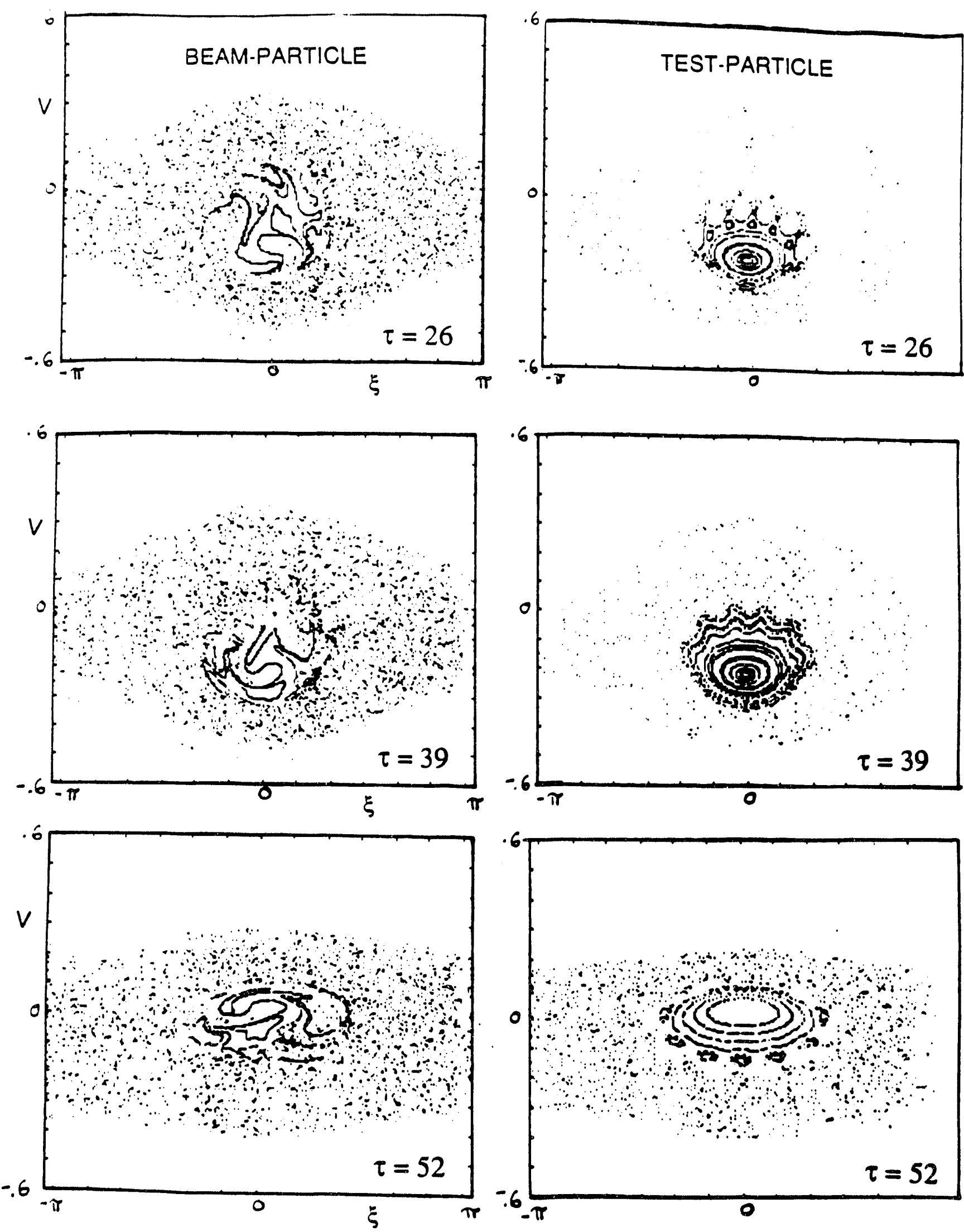

Fig. 6 

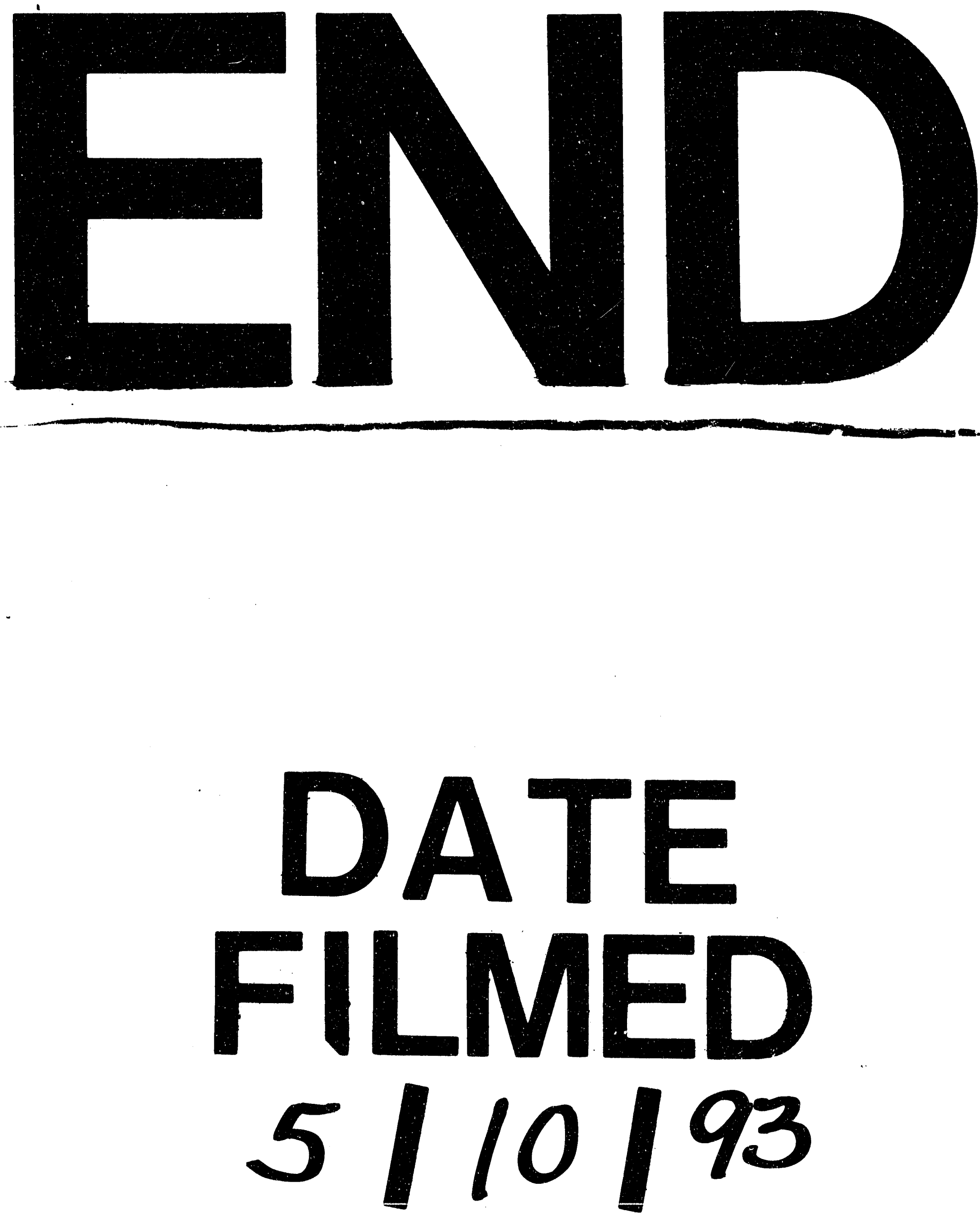
1 